\title{
The Effect of a Hypocaloric Diet Containing Olive Oil on Hepatic Steatosis Grading Using Tissue Elastography: A Randomized Controlled Trial
}

\author{
Wafaa Keshk ${ }^{1}$, Dina Ziada $^{2}$, Samah Soliman ${ }^{2}$, Ferial EL-Kalla ${ }^{2}$ \\ ${ }^{1}$ Department of Tropical Medicine and Infectious Diseases, Mahalla Fever Hospital, \\ Elmahalla Elkopra, Egypt. \\ ${ }^{2}$ Department Tropical Medicine and Infectious Diseases, Faculty of \\ Medicine, Tanta University, Tanta, Egypt
}

Corresponding Author Samah Mosaad Soliman.

Mobile: +2-01288226394

E mail: samah.soliman@ med.tanta.edu.eg

Key words: Hypocaloric diet, Olive Oil, Hepatic Steatosis Grading, Tissue Elastography.
Background and aim: The amount and type of dietary fat can affect fatty infiltration and lipid peroxidation in MAFLD. The beneficial effects of the olive oil, which is rich in monounsaturated fatty acids(MUFAs), on the hepatic fat content can be explained by the more rapid oxidization of the MUFAs than the saturated fatty acids in the postprandial phase. The aim of our study was to evaluate the effect of a hypocaloric diet containing olive oil on hepatic steatosis grading using tissue elastography controlled attenuation parameter (CAP).

Patients and Methods: This study was conducted on 58 patients with hepatic

\section{INTRODUCTION}

Metabolic-associated fatty liver disease (MAFLD), formly known as non-alcoholic fatty liver disease (NAFLD)is the most common chronic liver disease worldwide it is known as presence of hepatic steatosis in the absence of any secondary causes of hepatic fat accumulation [1].

In a high proportion of cases, MAFLD is related to "metabolic syndrome" (hyperglycemia, dyslipidemia, high blood pressure, and abdominal obesity) [1].

Peripheral insulin resistance affects carbohydrate and fat metabolism resulting in triglyceride (TG) accumulation in the liver. Resistance to insulin stimulation of glucose uptake via glucose transporter-4 by skeletal muscle and adipose tissue, in conjunction with the inhibition of lipolysis in adipose tissue by insulin, steatosis who were divided into Group 1: 30 patients on a hypocaloric diet that includes olive oil and group 2: 28 patients on a hypocaloric diet that does not include olive oil.

Results: Six months after the patients started a hypocaloric diet containing olive oil, there was a significant decrease in hepatic steatosis grading by CAP, as well as a significant decrease in serum triglyceride and cholesterol levels.

Conclusion: A hypocaloric diet containing olive oil has an important role in treatment of hepatic steatosis as shown by decrease of the grading by tissue elastography (CAP).

redirects glucose to the liver where the insulin continues to stimulate de novo lipogenesis and increase the flow of fatty acids from adipose tissue to the liver. This lead to increase consentration of TG in the liver. It is unclear how impairment in lipid export via very low density lipoprotein (VLDL) secretion, $\beta$ oxidation of free fatty acids (FFAs), or other metabolic pathways results in an inability to maintain fat balance, which leads to the development of fatty liver [2].

Dietary factors are thought to play a significant role in the development of MAFLD. The amount and type of dietary fat seem to affect fatty infiltration and lipid peroxidation in MAFLD, yet there is limited information on the effects of the type of dietary fat in MAFLD., the Mediterranean diet has recently gained attention as a diet that protect 
against MAFLD and cardiovascular disease. we known that olive oil is rich in monounsaturated fatty acids (MUFAs), olive oil responsible for the major part of the beneficial effects of the Mediterranean diet [3].

Hepatic steatosis can be assessed by using the Fibro Scan ${ }^{\circledR}$ device with the controlled attenuation parameter (CAP) facility. Ultrasound signals are attenuated by liver fat which can be measured using a conventional probe, giving a value between 100 and $400 \mathrm{~dB} / \mathrm{m}$ [4].

The purpose of study was to determine the effect of a hypocaloric diet containing olive oil on hepatic steatosis grading using tissue elastography controlled attenuation parameter (CAP).

\section{Patients AND METHODS}

This study was designed as a prospective open labelled randomized clinical trial. It was conducted at the Department of Tropical Medicine and Infectious diseases in Tanta University hospital from October 2018 to November 2019. Seventy- six patients with hepatic steatosis were enrolled, of these, 60 patients were randomly divided into two groups: Group 1: 30 patients with hepatic steatosis on a hypocaloric diet including olive oil and Group 2: 30 patients with hepatic steatosis on an olive oilfree hypocaloric diet. Two patients in group 2 dropped out during follow up ( figure 1). The two groups were matched as regard physical activity and caffeine intake ( 1 cup /day).

Patients included in the study were 18 years of age or older, capable of giving an informed consent, with a BMI of $30-40 \mathrm{~kg} / \mathrm{m} 2$ and diagnosed with hepatic steatosis by transient elastography (fibroscan®) (CAP) (>222 dB/m).

None of the patients took mineral or multivitamin supplements or lipid reduction medications within 3 months before the study was included or consumed olive products last month before the trial was included.

Exclusion criteria: Patients with fibrosis, cirrhosis, alcoholic steatohepatitis, or increased liver enzymes, as well as patients with secondary causes of hepatic fat buildup (heavy alcohol intake). The study excluded individuals with liver diseases that cause steatosis, such as hepatitis $\mathrm{C}$ or $\mathrm{B}$, Wilson disease, starvation, total parenteral nutrition, or bariatric surgery, as well as those taking specific medications (amiodarone, methotrexate, tamoxifen, corticosteroids, valproate, antiretroviral agents, or oral contraceptives). Individuals with diabetes mellitus or impaired glucose tolerance, hypothyroidism, pregnancy, breastfeeding, or who had a recent weight loss during the preceding six months were also excluded from the study.

At each visit, complete measurements were taken (height, weight, mid-arm circumference, waist circumference, and BMI) (at baseline, 3 months and 6 months from the beginning).

Determination of total daily calorie needs for weight loss: The total calories required to maintain weight for each patient was calculated by multiplying the appropriate activity factor by the basal metabolic rate(BMR) using the Harris Benedict Equation

For men $:$ BMR $=10 \mathrm{x}$ weight $(\mathrm{kg})+6.25 \mathrm{x}$ height $(\mathrm{cm})-5 \mathrm{x}$ age +5

For women $:$ BMR $=10 \mathrm{x}$ weight $(\mathrm{kg})+6.25 \mathrm{x}$ height $(\mathrm{cm})-5 \mathrm{x}$ age -161 . The required calories for maintenance decreased by 500 calories [5].

\section{Determination of total daily amount of olive} oil in the diet for patients from group 1:

Olive oil amount was calculated for each patient to provide $20 \%$ of the daily calories, considering that each tablespoon of olive oil (14g) gives 120 $\mathrm{kcal}$. The remaining $10 \%$ of daily fat calories were obtained from various sources, in keeping with the Mediterranean diet's emphasis on unsaturated fat, which accounts for around 30\% of total calories. Each patient is prescribed a balanced eating plan that is unique to them. Patients were advised to take the olive oil first thing in the morning or to incorporate it into their salad.

The BMI, calorie requirements, and daily consumption of olive oil were re-calculated at each follow up, and the patient was given a new diet plan.

In group 11 patients were received $10 \%$ of total fat as MUFA, $10 \%$ as PUFA , 9\% saturated fat and $1 \%$ transfat.

The 2 groups were matched as regard percent of macronutrients. They received $50 \%$ carbohydrate and $20 \%$ protein.

There is weight reduction programs by diet modification 
There is no any MAFLD affecting drugs as vitamin e, obeticholic acid, steroids, etc. used during the study

Patients were advised to walk for an average 20$30 \mathrm{~min} /$ day

\section{Techniques of transient elastography (FibroScan ${ }^{\mathrm{TM}}$ ):}

Patients were instructed to fast for at least four hours before examination (since liver stiffness increases significantly following food intake and acoustic radiation force impulse (ARFI) measures are best to be performed under fasting conditions).

To facilitate visualization of the liver's right lobe, patients were asked to lie either in the $30^{\circ}$ lateral left or supine position. The right arm was raised above the head to increase the intercostal spaces, and patients were instructed to breathe calmly as deep inspiration and expiration results in an erroneous rise in liver rigidity due to increased central venous pressure.

Liver stiffness was calculated automatically in the selected area, the average of multiple measurements (at least 10 valid measurements) were obtained, and reported with the following criteria; ratio of number of valid measurements to the total number of measurements is $\geq 60 \%$ and the interquartile range (IQR), which reflects the variability of measurements, is less than $30 \%$ of the median value of liver stiffness measurements.

Examiner used the gel-covered convex ultrasonic elastographic probe in the sixth, seventh, and eighth intercostal spaces of the right liver, as well as the elastography image and quantification box $2-6 \mathrm{~cm}$ beneath the liver capsule. We must to confirm that no major blood vessels, gallbladder, or bile ducts were present in the examined area.

\section{Statistical Analysis}

Statistical analysis of the present study was done by SPSS V26 (IBM Inc., Chicago,IL,USA)

quantitative variables were presented as mean and standard deviation(SD) and compared between the two groups utilizing unpaired Student's t- test. Repeated measures ANOVA was used to compare measurements compared to baseline. Qualitative variables were presented as frequency and percentage and were analyzed utilizing Chi- square test or fisher's exact test when appropriate. A two tailed $\mathrm{P}$ value $<0.05$ was considered statistically significant.

\section{RESULTS:}

A total number of 76 patients with hepatic steatosis were enrolled in this study and of these patients, 60 patients were randomized to receive either a hypocaloric diet including olive oil or an olive oil-free hypocaloric diet.

Demographic baseline characteristics of patients of the studied groups were showed in (Table 1)

BMI showed an insignificant difference between both groups at baseline and after 3 months $(\mathrm{P}=$ 0.481 and 0.145 ) while after 6 months, there was a significant decrease in group 1 than in group 2 $(\mathrm{P}=0.037)($ Table 2$)$

Waist circumference in group 1 and 2 decreased significantly at 6 months $(\mathrm{P}<0.001)$ with no significant difference between both groups at baseline, after 3 months and after 6 months $(\mathrm{P}=$ $0.102,0.286$ and 0.512 ).

Mid-arm circumference in group 1 decreased significantly at 3 and 6 months $(P=0.043$ and $<0.001$ ) while in group 2, it decreased insignificantly at 3 months but decreased significantly at 6 months $(\mathrm{P}<0.001)$. There was an insignificant difference between both groups at baseline, after 3 months and after 6 months (P $=0.269,0.804$ and 0.413$)$. (Table 3 )

Serum triglycerides in groups 1 and 2 decreased insignificantly at 3 months but at 6 months the decrease was statistically significant in group 1 $(P=0.006)$ while in group 2 it remained insignificant. However, on comparison between the two groups regarding serum triglyceride levels at baseline, 3 months and 6 months from starting the diet there was no significant difference $(\mathrm{P}=0.756, \quad 0.874$ and 0.248 respectively) (table 5)

Serum cholesterol decreased insignificantly 3 months into the study among patients of both groups I and 2, but at 6 months, the cholesterol levels of group 1 decreased significantly (P $<0.001)$ compared to the baseline levels. On the other hand, serum cholesterol in group 2 showed an insignificant difference at 6 months.

The difference in serum cholesterol levels between the patients from both groups was insignificant at baseline and after 3 months $(\mathrm{P}=$ 0.343 and 0.487 ) while after 6 months, the levels were significantly lower in group $1(\mathrm{P}=0.017)$. (table 5) 
HDL in group 1 decreased significantly at 3 and 6 months $(\mathrm{P}=0.043$ and $<0.001)$ while in group 2 , it showed an insignificant difference at 3 and 6 months compared to baseline.

CAP in group 1 and 2 decreased insignificantly at 3 months but became significantly lower at 6 months ( $\mathrm{P}<0.001)$. CAP showed an insignificant difference between both groups at baseline and after 3 months $(\mathrm{P}=0.906$ and 0.120$)$, while after 6 months, there was a more significant decrease in group 1 than group $2(\mathrm{P}=0.017)$. The difference between baseline CAP measurements and at 6 months was significantly more in group 1 than group $2(\mathrm{P}=0.012)$ (Table 4$)$

Table (1): Baseline characteristics of patients of the studied groups.

\begin{tabular}{|c|c|c|c|c|}
\hline \multicolumn{2}{|c|}{ Baseline patient characteristics } & \multirow{3}{*}{$\begin{array}{c}\begin{array}{c}\text { Group 1 } \\
(\mathbf{n}=\mathbf{3 0})\end{array} \\
45.43 \\
10.39\end{array}$} & \multirow{2}{*}{$\begin{array}{c}\begin{array}{c}\text { Group 2 } \\
(\mathbf{n}=\mathbf{2 8})\end{array} \\
40.29 \\
\end{array}$} & \multirow{3}{*}{$\begin{array}{r}\text { P value } \\
0.071\end{array}$} \\
\hline Age & Mean & & & \\
\hline & \pm SD & & 10.89 & \\
\hline \multirow[t]{2}{*}{ Sex } & Male & $5(17 \%)$ & $6(21 \%)$ & \multirow{2}{*}{0.945} \\
\hline & Female & $25(83 \%)$ & $22(79 \%)$ & \\
\hline \multirow[t]{2}{*}{ BMI } & Mean & 35.17 & 35.92 & \multirow[t]{2}{*}{0.481} \\
\hline & \pm SD & 4.15 & 3.88 & \\
\hline \multirow[t]{2}{*}{ Waist circumference } & Mean & 107.90 & 101.36 & \multirow[t]{2}{*}{0.102} \\
\hline & \pm SD & 14.99 & 14.96 & \\
\hline \multirow[t]{2}{*}{ Mid arm circumference } & Mean & 33.67 & 32.07 & \multirow[t]{2}{*}{0.269} \\
\hline & \pm SD & 5.94 & 4.84 & \\
\hline \multirow[t]{2}{*}{ Hemoglobin $(\mathrm{gm} / \mathrm{dL})$} & Mean & 11.61 & 11.45 & \multirow[t]{2}{*}{0.503} \\
\hline & \pm SD & 0.84 & 0.97 & \\
\hline \multirow[t]{2}{*}{ Platelets $\left(* 10^{3} / \mathbf{m m}^{3}\right)$} & Mean & 248.50 & 271.00 & \multirow[t]{2}{*}{0.072} \\
\hline & \pm SD & 52.18 & 39.86 & \\
\hline \multirow[t]{2}{*}{ Total Leucocyte Count $\left(* 10^{3} / \mathrm{mm}^{3}\right)$} & Mean & 5.65 & 6.14 & \multirow[t]{2}{*}{0.089} \\
\hline & \pm SD & 0.81 & 1.30 & \\
\hline \multirow[t]{2}{*}{ Serum creatinine $(\mathrm{mg} / \mathrm{dL})$} & Mean & 0.97 & 0.98 & \multirow[t]{2}{*}{0.694} \\
\hline & \pm SD & 0.11 & 0.12 & \\
\hline \multirow[t]{2}{*}{ Blood urea (mg/dL) } & Mean & 22.87 & 21.57 & \multirow[t]{2}{*}{0.072} \\
\hline & \pm SD & 2.22 & 3.11 & \\
\hline \multirow{2}{*}{$\begin{array}{c}\text { Total serum bilirubin } \\
(\mathbf{m g} / \mathbf{d L})\end{array}$} & Mean & 0.98 & 0.97 & \multirow[t]{2}{*}{0.561} \\
\hline & \pm SD & 0.09 & 0.06 & \\
\hline \multirow{2}{*}{$\begin{array}{c}\text { Alanine aminotransferase (ALT) } \\
(\mathrm{U} / \mathbf{L})\end{array}$} & Mean & 26.11 & 23.14 & \multirow[t]{2}{*}{0.102} \\
\hline & \pm SD & 7.86 & 5.46 & \\
\hline \multirow{2}{*}{$\begin{array}{c}\text { Aspartate aminotransferase (AST) } \\
\text { )U/L }\end{array}$} & Mean & 24.53 & 22.69 & \multirow[t]{2}{*}{0.384} \\
\hline & \pm SD & 10.01 & 5.05 & \\
\hline Serum albumin & Mean & 3.89 & 3.96 & 0.549 \\
\hline$(\mathrm{gm} / \mathbf{d L})$ & \pm SD & 0.43 & 0.37 & \\
\hline Serum triglycerides & Mean & 130.13 & 126.0 & 0.756 \\
\hline$(\mathrm{mg} / \mathrm{dL})$ & \pm SD & 54.62 & 45.21 & \\
\hline Serum cholesterol & Mean & 203.13 & 193.5 & 0.343 \\
\hline$(\mathrm{mg} / \mathrm{dL})$ & \pm SD & 42.89 & 32.7 & \\
\hline Low density lipoprotein (LDL) & Mean & 125.15 & 122.6 & 0.775 \\
\hline$(\mathrm{mg} / \mathrm{dL})$ & \pm SD & 41.0 & 42.02 & \\
\hline High density lipoprotein (HDL) & Mean & 49.7 & 47.05 & 0.094 \\
\hline$(\mathrm{mg} / \mathrm{dL})$ & \pm SD & 7.33 & 3.89 & \\
\hline
\end{tabular}


Table (2): Body mass index (BMI) $(\mathrm{kg} / \mathrm{m} 2)$ in both groups at baseline, 3 and 6 months of inclusion in the study.

\begin{tabular}{|c|c|c|c|c|}
\hline & & Baseline & 3 mon & 6 mon \\
\hline \multirow{3}{*}{$\begin{array}{c}\text { Group 1 } \\
(n=30) \\
\text { With olive oil }\end{array}$} & Mean & 35.17 & 33.62 & 31.96 \\
\hline & \pm SD & 4.15 & 4.09 & 3.96 \\
\hline & \multicolumn{2}{|c|}{ P1 } & 0.152 & $<0.001 *$ \\
\hline \multirow{3}{*}{$\begin{array}{c}\text { Group 2 } \\
(n=28) \\
\text { Without olive oil }\end{array}$} & Mean & 35.92 & 35.17 & 34.16 \\
\hline & \pm SD & 3.88 & 3.88 & 3.86 \\
\hline & \multicolumn{2}{|c|}{ P2 } & 0.478 & $<0.001 *$ \\
\hline \multicolumn{2}{|c|}{ P value } & $\mathrm{P} 3=0.481$ & $\mathrm{P} 4=0.145$ & P5 $=0.037 *$ \\
\hline
\end{tabular}

P1: P value in group 1 at 3 months compared to "baseline"; P2: P value in group 2 at six months compared to the baseline; P3: Comparison between both groups at baseline; P4: Comparison between both groups at 3 months; P5: Comparison between both groups at 6 months, *significant as p value $<0.05$

Table (3): Waist circumference $(\mathrm{cm})$ and mid-arm circumference $(\mathrm{cm})$ in patients from both groups.

\begin{tabular}{|c|c|c|c|c|}
\hline \multicolumn{2}{|c|}{ Waist circumference $(\mathrm{cm})$ in both groups } & Baseline & 3 months & 6 months \\
\hline \multirow{3}{*}{$\begin{array}{l}\text { Group } 1 \\
(\mathbf{n}=\mathbf{3 0})\end{array}$} & Mean & 107.90 & 103.80 & 100.07 \\
\hline & $\pm \mathrm{SD}$ & 14.99 & 14.71 & 13.97 \\
\hline & \multicolumn{2}{|c|}{$\mathrm{P} 1$} & 0.289 & $<0.001 *$ \\
\hline \multirow{3}{*}{$\begin{array}{l}\text { Group } 2 \\
(n=28)\end{array}$} & Mean & 101.36 & 99.64 & 97.61 \\
\hline & $\pm \mathrm{SD}$ & 14.96 & 14.69 & 14.42 \\
\hline & \multicolumn{2}{|c|}{$\mathrm{P} 2$} & 0.667 & $<0.001 *$ \\
\hline \multicolumn{2}{|l|}{ P value } & $\mathrm{P} 3=0.102$ & $\mathrm{P} 4=0.286$ & $\mathrm{P} 5=0.512$ \\
\hline \multicolumn{2}{|c|}{ Mid-arm circumference $(\mathrm{cm})$ in both groups } & Baseline & 3 mon & 6 mon \\
\hline \multirow{3}{*}{$\begin{array}{l}\text { Group } 1 \\
(n=30)\end{array}$} & Mean & 33.67 & 30.63 & 28.27 \\
\hline & $\pm \mathrm{SD}$ & 5.94 & 5.43 & 5.04 \\
\hline & \multicolumn{2}{|c|}{$\mathrm{P} 1$} & $0.043 *$ & $<0.001 *$ \\
\hline \multirow{4}{*}{$\begin{array}{l}\text { Group } 2 \\
(n=28)\end{array}$} & Mean & 32.07 & 30.96 & 29.29 \\
\hline & $\pm \mathrm{SD}$ & 4.84 & 4.64 & 4.31 \\
\hline & \multicolumn{2}{|c|}{$\mathrm{P} 2$} & 0.386 & $<0.001 *$ \\
\hline & \multicolumn{2}{|c|}{$\mathrm{P}$ value } & $\mathrm{P} 3=0.269$ & $\mathrm{P} 4=0.804$ \\
\hline
\end{tabular}

P1: P value in group 1 at 3 months compared to "baseline"; P2: P value in group 2 at six months compared to the baseline; P3: Comparison between both groups at baseline; P4: Comparison between both groups at 3 months; P5: Comparison between both groups at 6 months, *significant as p value $<0.05$.

Table (4): Controlled attenuation parameter (CAP) $(\mathrm{dB} / \mathrm{m})$ in both groups.

\begin{tabular}{|c|c|c|c|c|c|}
\hline & & Baseline & 3 mon & $6 \mathrm{mon}$ & Difference \\
\hline \multirow{3}{*}{$\begin{array}{l}\text { Group 1 } \\
(\mathbf{n}=\mathbf{3 0})\end{array}$} & Mean & 306.67 & 284.67 & 257.33 & -49.33 \\
\hline & $\pm \mathrm{SD}$ & 47.12 & 60.01 & 56.78 & 9.66 \\
\hline & \multicolumn{2}{|c|}{$\mathrm{P} 1$} & 0.120 & $<0.001 *$ & \\
\hline \multirow{3}{*}{$\begin{array}{c}\text { Group } 2 \\
(n=28)\end{array}$} & Mean & 305.25 & 305.64 & 288.46 & -16.79 \\
\hline & $\pm \mathrm{SD}$ & 43.33 & 37.75 & 36.43 & 23.40 \\
\hline & \multicolumn{2}{|c|}{$\mathrm{P} 2$} & 0.971 & $<0.001 *$ & \\
\hline \multicolumn{2}{|c|}{$\mathrm{P}$ value } & $\mathrm{P} 3=0.906$ & $\mathrm{P} 4=0.120$ & $\mathrm{P} 5=0.017 *$ & $0.012 *$ \\
\hline
\end{tabular}

P1: P value in group 1 at 3 months compared to "baseline"; P2: P value in group 2 at six months compared to the baseline; P3: Comparison between both groups at baseline; P4: Comparison between both groups at 3 months; P5: Comparison between both groups at 6 months, *significant as $\mathrm{p}$ value $<0.05$. 
Table (5): Complete lipid profile in both groups.

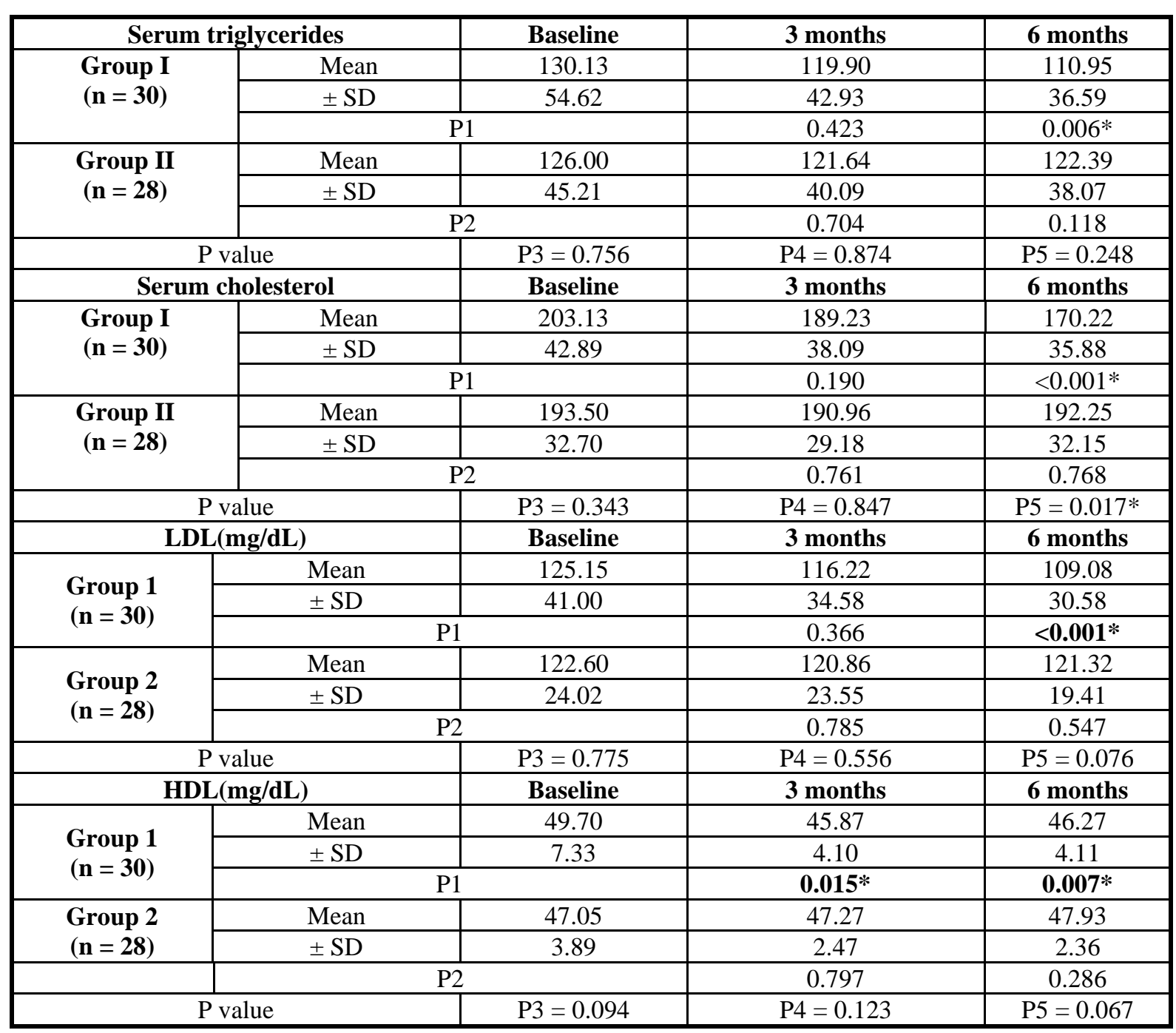

\section{DISCUSSION}

Dietary components, especially the type and the quantity of fats, are critical for liver fat accumulation and are account for $15 \%$ of the liver fat content. Dietary fat can contribute to liver steatosis directly or indirectly via the effect on adipose tissue [6].

The research undertaken in this field are limited to examining the effects of a high fat and MUFA-modified Mediterranean diet on MAFLD patients and comparing it to a low fat diet.

Numerous studies have established a link between reducing saturated fat intake and lowering total cholesterol, very low-density lipoprotein (LDL), and triglyceride levels in the blood [7].
According to Nakahara et al study, 37.5 percent and 19.5 percent of people with MAFLD, respectively, had hyper-LDL cholesterol and hypo-HDL cholesterol[8].

Additional possible mechanisms for cholesterol accumulation in MAFLD can be deduced from published data, most notably Min et al work. which examined the expression of cholesterol genes in MAFLD in detail. Reduced expression of the cholesterol efflux gene ABCG8 results in decreased FC efflux in fatty hepatocytes [9].

The current study demonstrates that a hypocaloric diet containing $20 \%$ of daily calories from olive oil resulted in weight loss along with significant reductions in total plasma cholesterol and serum triglyceride levels, whereas a hypocaloric diet containing no olive oil resulted in weight loss but no change in serum cholesterol 
or triglyceride levels. In line with our findings, a Garg meta-analysis of studies on diabetic individuals indicated that a high-fat diet containing between 22\% and 33\% of MUFA energy resulted in lower total plasma cholesterol than a low-fat, high-carbohydrate diet (49\% and $60 \%$ energy) [7].

Additionally, Erkkila et al. discovered that MUFAs those found in olive oil, almonds, and avocados have a beneficial effect on cardiovascular disease risk and lipid profile[10].

Also , Sofi et al.[11] discovered a significant improvement of liver enzymes and triglycerides in individuals who consumed MUFA-enriched olive oil (post: 132,8 63,7 vs. pre: 164,5 82,5 $\mathrm{mg} / \mathrm{dl} ; \mathrm{P}=0,04)$.

The lowering effect of the hypocaloric diet with olive oil on serum triglyceride levels in our study may be attributed to increased lipoprotein lipase fat activity, which results in increased clearance of circulating lipoproteins rich in triglycerides.

The beneficial effects of the MUFAs on the hepatic fat accumulation can be explained by the fact that MUFAs oxidize more rapidly than saturated fatty acids in the postprandial phase. The more beneficial MUFA deposits in adipose tissue rather than the liver may thus help avoid fat formation in the liver in response to a MUFArich diet. Additionally, a MUFA-rich diet increases lipoprotein lipase activity more than a saturated fat-rich diet, resulting in higher clearance of circulating lipoproteins with a high triglyceride content. Along with the type, fat amount plays a role in pathogenesis and, most likely, treatment of fatty liver [12].

The current study demonstrated a significant reduction in LDL and HDL cholesterol levels following a hypocaloric diet high in olive oil. Consumption of MUFA has been shown to reduce oxidised LDL in prior research[13]. Fito et al2017 .'s study indicated that both total HDL and LDL/HDL cholesterol levels reduced in both groups of patients who consumed olive oil or nuts as part of a Mediterranean diet[14]. Similarly, Sacks et al. and Williams et al. showed that LDL cholesterol and TG concentrations decreased without a corresponding fall in HDL [15,16].

Julius, on the other hand, revealed that substituting MUFAs for carbohydrate and saturated fat led to an increase in HDL, however their study population differed as it included diabetic patients [17].

A negative relationship between MUFA consumption and the progression of MAFLD has been reported. In addition, the consumption of $20 \mathrm{~g} /$ day for 12 weeks in hypocaloric diets attenuated the degree of fatty liver in patients with MAFLD[18].

The hypocaloric, high MUFA diet demonstrated a significant reduction in the liver fat content as determined by CAP $(\mathrm{P}<0.001)$ in patients with MAFLD in the current study. The beneficial effects of MUFAs on the fat accumulation of the liver can be explained by the fact that MUFAs are more rapidly oxidized in the postprandial phase than saturated fatty acids. Olive oil contains a high concentration of monounsaturated fatty acids, especially oleic acids, as well as phenolic substances such as oleuropin and hydroxytyrosol. Because MUFAs are prone to adipose tissue deposition rather than hepatic deposition, a diet rich in MUFAs can help avoid liver deposition and alleviate present steatosis. A MUFA-rich diet may help reduce liver fat by improving the cross-talk between adipose tissue and hepatic metabolism via the regulation of adipokines and inflammatory marker Synthesis [19].

Olive oil also protects the liver by inhibiting hepatocyte ballooning, fibrogenesis, and lipid peroxidation by inhibiting inflammatory signalling pathways, oxidative stress, endoplasmic reticulum stress, mitochondrial dysfunction, and insulin resistance. Olive oil exerts these molecular hepatoprotective effects by activating an erythroid-derived nuclear transcription factor of 2-like (Nfr2) and inducing a cellular antioxidant response; inactivating a nuclear transcription factor B (NF); preventing a cellular inflammatory response; and inhibiting PERK pathways, thereby preventing endoplasmic reticular stress, autophagy, and other cellular processes [20]. Olive oil's multiple benefits can be utilised to prevent or reverse liver disease.

The hypocaloric diet, which included olive oil, took time to have a beneficial effect on the blood lipid profile and liver fat level, with significant changes occurring after six months after dietary start. This demonstrates that diets high in MUFAs may be more beneficial on the long run. 
Our study was limited by being a single center study on a small number of patients and that the period of study was not extended to more than six months.

\section{Recommendations:}

A hypocaloric diet containing olive oil should be used in the treatment of hepatic steatosis and dyslipidemia. Further multi-center studies with larger sample sizes, and different populations are needed to generalize the results of our research. Studies with longer follow-up of 1 year or 5 years are also recommended to evaluate the effects of long-term use of olive oil on liver steatosis and dyslipidemia and whether the effect is sustained.

\section{Acknowledgement: Non}

Funding information: None. Author funded

Conflict of Interest: "The authors have no conflict of interest to report".

Ethical considerations: A written informed consent was obtained from all participants in the study.The work was done according to the declaration of Helsinki and the sound practices and was approved by the Ethical Committee of Faculty of Medicine , Tanta university. (Approval code 32496/ 07/18).

\section{RESEARCH HIGHLIGHTS}

1- Non-alcoholic fatty liver disease (NAFLD) is the most common chronic liver disease worldwide.

2- Currently there are no optimal therapy for NAFLD.

3- Dietary factors are presumed to play a key role in the progression of NAFLD.

4- The present study revealed that a hypocaloric diet including olive oil at an amount providing $20 \%$ of the daily calories produced a weight loss accompanied by significant lowering of plasma total cholesterol and serum triglyceride levels.

5- The effect of the hypocaloric, high MUFA diet in the current study proved a significantly beneficial lowering effect on liver fat content in patients with NAFLD as estimated by CAP.

\section{REFERENCES}

1. Chalasani N, Younossi Z, Lavine JE, Diehl, AM, Brunt EM, Cusi K, et al. The diagnosis and management of non-alcoholic fatty liver disease: practice Guideline by the American Association for the Study of Liver Diseases, American College of Gastroenterology, and the American Gastroenterological Association. Hepatology 2012; 55:2005-23.

2. Postic C, Girard J. Contribution of de novo fatty acid synthesis to hepatic steatosis and insulin resistance: lessons from genetically engineered mice. J Clin Invest 2008; 118:829-38.

3. Biolato M, Manca F, Marrone G, Cefalo C, Racco S, Miggiano GA, et al. Intestinal permeability after Mediterranean diet and low-fat diet in non-alcoholic fatty liver disease. World $J$ Gastroenterol 2019; 25:509-520.

4. Sasso M, Beaugrand M, de Ledinghen V, Douvin C, Marcellin P, Poupon R, et al. Controlled attenuation parameter (CAP): a novel VCTE guided ultrasonic attenuation measurement for the evaluation of hepatic steatosis: preliminary study and validation in a cohort of patients with chronic liver disease from various causes. Ultrasound Med Biol 2010; 36:1825-35.

5. Seagle HM, Strain GW, Makris A, Reeves RS, Position of the American Dietetic Association: weight management. $J \quad A m$ Diet Assoc.2009;109:330-346.

6. Donnelly KL, Smith CI, Schwarzenberg SJ, Jessurun J, Boldt MD, Parks EJ. "Sources of fatty acids stored in liver and secreted via lipoproteins in patients with nonalcoholic fatty liver disease," The Journal of Clinical Investigation 2005; 115 (5): 1343-1351.

7. Garg A. High-monounsaturated-fat diets for patients with diabetes mellitus: a meta-analysis. Am J Clin Nutr, 1998; 67:577s-582s.

8. Nakahara T, Hyogo H, Yoneda M, Sumida Y, Eguchi Y, Fujii H, et al. Japan Study Group of Nonalcoholic Fatty Liver Disease (JSG-NAFLD) Type 2 diabetes mellitus is associated with the fibrosis severity in patients with nonalcoholic fatty liver disease in a large retrospective cohort of Japanese patients. J Gastroenterol 2013; 49(11):1477-84.

9. Min HK, Kapoor A, Fuchs M, Mirshahi F, Zhou H, Maher J, Kellum J, Warnick R, Contos MJ, Sanyal AJ. Increased hepatic synthesis and dysregulation of cholesterol metabolism is associated with the severity of nonalcoholic fatty liver disease. Cell Metab 2012; 15:665-674. 
10. Erkkila AT, Matthan NR, Herrington DM, Lichtenstein AH. Higher plasma docosahexaenoic acid is associated with reduced progression of coronary atherosclerosis in women with CAD. J Lipid Res 2006; 47:2814-9.

11. Sofi F, Giangrandi I, Cesari F, Corsani I, Abbate R, Gensini GF, et al. 2010. Effects of a 1-year dietary intervention with $\mathrm{n}-3$ polyunsaturated fatty acid-enriched olive oil on non-alcoholic fatty liver disease patients: a preliminary study. Int J Food Sci Nutr 2010; 61:792-802.

12. Rivellese AA, Giacco R, Annuzzi G, De Natale C, Patti L, Di Marino L, et al. Effects of monounsaturated vs. saturated fat on postprandial lipemia and adipose tissue lipases in type 2 diabetes. Clinical Nutrition 2008; 27:133-141.

13. Lapointe A, Couillard C, Lemieux S. Effects of dietary factors on oxidation of low-density lipoprotein particles. J Nutr Biochem 2006; 17:645-58

14. Fito M, Guxens M, Corella D, Saez G, Estruch R, de la Torre R, et al. Effect of a traditional Mediterranean diet on lipoprotein oxidation: a randomized controlled trial. Arch Intern Med 2007; 167:1195-1203.

15. Sacks FM. Dietary fat, the Mediterranean diet, and health: reports from scientific exchanges, 1998 and 2000. Introduction. Am J Med 2002; 113 Suppl 9B, 1s-4s.
16. Williams CM. Beneficial nutritional properties of olive oil: implications for postprandial lipoproteins and factor VII. Nutr Metab Cardiovasc Dis 2001; 11:51-6.

17. Julius U. Influence of plasma free fatty acids on lipoprotein synthesis and diabetic dyslipidemia. Exp Clin Endocrinol Diabetes 2003; 111:246-50.

18. Rezaei S, Akhlaghi M, Sasani MR, Barati Boldaji R. Olive oil lessened fatty liver severity independent of cardiometabolic corrections in patients with non-alcoholic fatty liver disease: a randomized clinical trial. Nutrition 2019;57:154161.

19. Bozzetto L., Prinster A., Annuzzi G., Costagliola L., Mangione A., Vitelli A., Mazzarella R., Longobardo M., Mancini M., Vigorito C., et al. Liver fat is reduced by an isoenergetic mufa diet in a controlled randomized study in type 2 diabetic patients. Diabetes Care. 2012;35:14291435. doi: 10.2337/dc12-0033

20. Soto-Alarcon SA, Valenzuela R, Valenzuela A, Videla LA. Liver Protective Effects of Extra Virgin Olive Oil: Interaction Between Its Chemical Composition and the Cell-signaling Pathways Involved in Protection Affiliations expand. Endocrine, Metabolic \& Immune Disorders Drug Targets 2018; 18(1):758. 